\title{
Sliding window haplotype approaches overcome single SNP analysis limitations in identifying genes for meat tenderness in Nelore cattle
}

Camila U. Braz ${ }^{1 *}$ D, Jeremy F. Taylor ${ }^{2}$, Tiago Bresolin ${ }^{1}$, Rafael Espigolan ${ }^{1}$, Fabieli L. B. Feitosa ${ }^{1}$, Roberto Carvalheiro ${ }^{1}$, Fernando Baldi ${ }^{1}$, Lucia G. de Albuquerque ${ }^{1}$ and Henrique N. de Oliveira ${ }^{* *}$

\begin{abstract}
Background: Traditional single nucleotide polymorphism (SNP) genome-wide association analysis (GWAA) can be inefficient because single SNPs provide limited genetic information about genomic regions. On the other hand, using haplotypes in the statistical analysis may increase the extent of linkage disequilibrium (LD) between haplotypes and causal variants and may also potentially capture epistastic interactions between variants within a haplotyped locus, providing an increase in the power and robustness of the association studies. We performed GWAA (413,355 SNP markers) using haplotypes based on variable-sized sliding windows and compared the results to a single-SNP GWAA using Warner-Bratzler shear force measured in the longissimus thorasis muscle of 3161 Nelore bulls to ascertain the optimal window size for identifying the genomic regions that influence meat tenderness.

Results: The GWAA using single SNPs identified eight variants influencing meat tenderness on BTA 3, 4, 9, 10 and 11. However, thirty-three putative meat tenderness QTL were detected on BTA 1, 3, 4, 5, 8, 9, 10, 11, 15, 17, 18, 24, 25, 26 and 29 using variable-sized sliding haplotype windows. Analyses using sliding window haplotypes of 3, 5, 7, 9 and 11 SNPs identified 57, 61, 42, 39, and 21\% of all thirty-three putative QTL regions, respectively; however, the analyses using the 3 and 5 SNP haplotypes, cumulatively detected $88 \%$ of the putative QTL. The genes associated with variation in meat tenderness participate in myogenesis, neurogenesis, lipid and fatty acid metabolism and skeletal muscle structure or composition processes.
\end{abstract}

Conclusions: GWAA using haplotypes based on variable-sized sliding windows allowed the detection of more QTL than traditional single-SNP GWAA. Analyses using smaller haplotypes (3 and 5 SNPs) detected a higher proportion of the putative QTL.

Keywords: Additive genetic variance, Beef cattle, Haplotype, GWAA, Meat tenderness

\section{Background}

Following the completion of the first draft bovine reference genome assembly, a high-density single nucleotide polymorphism (SNP) genotyping assay was developed [1], enabling genome-wide association analyses (GWAA), which are useful in understanding the underlying biology of traits $[2,3]$. Several GWAA have identified SNP associated with

\footnotetext{
*Correspondence: camila_urbano@yahoo.com.br; oliveira.hn@gmail.com ${ }^{1}$ Animal Science Department, São Paulo State University (Unesp), Jaboticabal, SP 144884-900, Brazil

Full list of author information is available at the end of the article
}

meat tenderness in cattle [4-7], which is one of the most important attributes impacting consumer satisfaction and the price of beef [8]. Moreover, meat tenderness is the most important trait requiring improvement in Nelore catthe in order to increase beef quality and ensure consumer acceptability. However, studies have shown that the traditional single-SNP GWAA can be inefficient because single-SNPs provide limited information about the content of flanking genomic regions $[9,10]$. On the other hand, using haplotypes in the statistical analysis may increase the extent of linkage disequilibrium (LD) between haplotypes

(c) The Author(s). 2019 Open Access This article is distributed under the terms of the Creative Commons Attribution 4.0 International License (http://creativecommons.org/licenses/by/4.0/), which permits unrestricted use, distribution, and reproduction in any medium, provided you give appropriate credit to the original author(s) and the source, provide a link to the Creative Commons license, and indicate if changes were made. The Creative Commons Public Domain Dedication waiver (http://creativecommons.org/publicdomain/zero/1.0/) applies to the data made available in this article, unless otherwise stated. 
and causal variants and also potentially capture epistastic interactions between variants within a haplotyped locus, providing increased power and robustness for association studies [10-16].

The use of haplotypes in GWAA analysis has not been widely exploited because there is no consensus on how many adjacent SNPs should be haplotyped, or on what is the best methodology for the definition and analysis of the haplotype blocks [17]. Different criteria have been proposed, but the most widely used approach is based on the extent of LD between markers as described by [18], which combines SNPs into haplotype blocks in genomic regions of high LD, i.e., with low evidence for recombination. However, the use of this approach can result in "orphan" SNPs that fall outside of predefined LD blocks, leading to a loss in ability to dissect genetic variation in these regions in the association analysis [14]. Thus, haplotype block approaches may not be the most efficient approach for association studies [19]. An alternative strategy for performing haplotype-based association analyses is based on overlapping sliding windows, in which several neighboring contiguous SNPs are included in a haplotype analysis, spanning the entire genome regardless of the extent of LD between the markers $[10,14,20]$. This approach has been shown to be more powerful than single-SNP and LD block-based haplotype analyses, particularly in genomic regions with high recombination and low LD [10, 21, 22]. Furthermore, according to [10], the use of sliding window haplotype analysis increases the likelihood of QTL detection and identification of the genomic regions harboring the causal variants.

We performed a GWAA using haplotypes defined by variable-sized sliding windows and compared the results to single-SNP GWAA to ascertain the optimal window size for identifying QTL regions that influence meat tenderness in Nelore cattle. The results of this study provide a better understanding of the genetic basis of meat tenderness in zebu cattle.

\section{Methods}

\section{Animals}

The 3161 Nelore bulls used in this study were born between 2008 and 2013 and were sourced from three different animal breeding programs. These animals were raised on pasture, finished in a feedlot for approximately 90 days, and then slaughtered in commercial slaughterhouses at a mean age of $691 \pm 102$ days. Contemporary groups (CG) were defined by the combination of farm of origin, year of birth, management group as long-yearlings and month and year of slaughter. The animals belonged to 116 CG with at least three animals per group.

\section{Phenotypic and genotypic data}

The animals were slaughtered in commercial slaughter houses and the carcasses were identified by tags and chilled for 24 to $48 \mathrm{~h}$ post-mortem. Steaks of $2.54 \mathrm{~cm}$ thickness were then collected from the longissimus thoracis muscle between the 12th and 13th ribs from the left half of the carcasses. The steaks were vacuum sealed and aged in a cold chamber for $150 \mathrm{~h}$ at $1{ }^{\circ} \mathrm{C}$ and then were frozen at $-20^{\circ} \mathrm{C}$. Next, the steaks were cooked in an oven to an internal temperature of $71^{\circ} \mathrm{C}$ as proposed by [23]. Warner-Bratzler shear force (WBSF), a mechanical measurement of tenderness, was measured $24 \mathrm{~h}$ after cooking using a Salter shearing device (G-R Electric, Manhattan, KS). Eight $1.27 \mathrm{~mm}$ meat cores were obtained from each sample and the average shear force of the eight cores was used in analysis. The mean WBSF for the 3161 Nelore bulls was $5.9 \pm 1.80 \mathrm{~kg}$ varying from $1.6 \mathrm{~kg}$ to $11.9 \mathrm{~kg}$.

Using a DNeasy Blood \& Tissue Kit (Qiagen $\mathrm{GmbH}$, Hilden, Germany), tissues from the longissimus thoracis muscle were used to extract DNA according to the manufacturer's instructions. Genotyping was performed by high-density BeadArray technology $(777 \mathrm{~K})$ using the Illumina (San Diego, CA) BovineHD Infinium Assay with an Illumina HiScan System ${ }^{\circ}$ for 1405 animals and the 1756 remaining animals were genotyped with a lower density bead array (GGP75Ki). Genotypes were imputed to the content of the BovineHD assay and phased using FImpute software [24] including available pedigree information. The average imputation accuracy from GGP75Ki to Illumina BovineHD was $98.93 \%$, as reported by [25]. Samples for which the genotype call rate was less than $90 \%$ and SNP markers with a call rate of less than $95 \%$, or that had a minor allele frequency of less than 5\%, or Hardy Weinberg Equilibrium test statistic probability of less than $10^{-5}$ or that were unmapped to autosomes or sex-linked were removed. A total of 413,355 SNP markers remained for analysis. The genomic coordinates for each SNP marker were based on the Bos taurus UMD3.1 reference assembly.

\section{Construction of haplotypes}

Five different haplotype sizes were constructed based on overlapping sliding windows methodology spanning the entire genome: three (SW3), five (SW5), seven (SW7), nine (SW9) or eleven (SW11) SNPs. Given an ordered set of markers $\left(\mathrm{SNP}_{1}, \mathrm{SNP}_{2}, \mathrm{SNP}_{3}, \ldots, \mathrm{SNP}_{n}\right)$, where $n$ is the number of SNP markers on the chromosome, sliding windows of overlapping haplotypes are tested in sequence. i.e. for SW3: haplotype $1\left(\mathrm{SNP}_{1}-\mathrm{SNP}_{2}-\mathrm{SNP}_{3}\right)$, haplotype 2 $\left(\mathrm{SNP}_{2}-\mathrm{SNP}_{3}-\mathrm{SNP}_{4}\right)$, haplotype $3\left(\mathrm{SNP}_{3}-\mathrm{SNP}_{4}-\mathrm{SNP}_{5}\right), \ldots$ haplotype $n\left(\mathrm{SNP}_{n-2}, \mathrm{SNP}_{n-1}, \mathrm{SNP}_{n}\right)[14,20]$. The haplotypes were estimated for each locus and the diplotype of each animal was estimated as the combination of haplotypes $i$ and $i$, 
at locus $j$. Thus, the dummy variable for each haplotype were coded as: $0=$ no copies of the haplotype, $1=$ one copy of the haplotype (paternal or maternal), and $2=$ two copies of the haplotype (paternal and maternal).

\section{Genome-wide association analysis}

The WBSF was pre-adjusted for fixed effect of CG and age at slaughter as covariate (linear effect). Fixed effects were estimated using a single-trait animal model implemented in AIREMLF90 [26]. The univariate linear mixed model analysis was performed for pre-adjusted WBSF using single-SNPs and each size of haplotype (three, five, seven, nine or eleven SNPs) individually in GEMMA [27] using the model: $y=1 \mu+X \beta+Z u+e ; u \sim M V N_{n}(0$, $\left.G V_{g}\right) ; \quad e \sim M V N_{n}\left(0, I V_{e}\right)$; where: $y$ is an $n$-vector of pre-adjusted WBSF; $\mu$ is the overall mean; $X$ is the incidence matrix for single SNP or haplotype; $\beta$ is the single SNP allele or haplotype effects; $Z$ is an $n \times n$ identity matrix; $u$ is an $n$-vector of random residual additive genetic effects; $G$ is a $n \times n$ genomic relationship matrix (GRM); $e$ is a vector of residuals; $V_{g}$ is the residual additive genetic variance component; $V_{e}$ is the residual variance component; and $I$ is an $n \times n$ identity matrix. $M V N_{n}$ denotes the $n$-dimensional multivariate normal distribution. The GRM was estimated using the standardized genotypes for all 413,355 SNP markers retained for analysis following filtering, using GEMMA. The same GRM was used for the single-SNPs and haplotype-based association analyses.

\section{Estimating additive genetic variance}

The additive genetic variance $\left(\mathrm{V}_{\mathrm{A}}\right)$ explained by SNP markers was estimated as: $\sigma_{i}^{2}=2 p_{i}\left(1-p_{i}\right) a_{i}^{2}$, where: $\sigma_{i}^{2}$ is the additive genetic variance for the $i^{\text {th }} \mathrm{SNP} ; p_{i}$ is the allele frequency of one of the alleles at the $i^{\text {th }}$ SNP; and $a_{i}$ is the additive effect of the $i^{\text {th }}$ SNP. An equivalent equation was used to estimate $\mathrm{V}_{\mathrm{A}}$ explained by the haplotyped loci [28], as follows: $\sigma_{j}^{2}=\sum_{i=1}^{k_{j}-1} \sum_{l=2}^{k_{j}}\left(a_{i j}-a_{l j}\right)^{2} p_{i j} p_{l j}$, for $l>i$, where: $\sigma_{j}^{2}$ is the additive genetic variance for the $j^{\text {th }}$ haplotype, $a_{i j}$ is the additive effect of the $i^{\text {th }}$ allele at the $j^{\text {th }}$ haplotype, $a_{l j}$ is the additive effect of the $l^{\text {th }}$ allele at the $j^{\text {th }}$ haplotype, $p_{i j}$ is the allele frequency for the $i^{\text {th }}$ allele at the $j^{\text {th }}$ haplotype, $p_{l j}$ is the allele frequency for the $l^{\text {th }}$ allele at the $j^{\text {th }}$ haplotype, and $k_{j}$ is the number of existing alleles at the $j^{\text {th }}$ haplotype. The additive effects and frequencies of the SNP and haplotype alleles were calculated using GEMMA software as described in section "Genome-wide association analysis".

For the identification of the SNPs and haplotyped loci that explained the greatest amounts of $\mathrm{V}_{\mathrm{A}}$ for WBSF, the estimated $V_{A}$ were assumed to follow a gamma distribution with parameters shape $(\alpha)$ and rate $(\beta)$ [29]. The parameters $\left(\alpha\right.$ and $\beta$ ) were predicted using the $V_{A}$ explained by the SNPs and haplotyped loci which allowed establishing the value of gamma distribution quantile corrected for multiple testing by Bonferroni method $(\alpha \leq 0.05)$. For the SNPs, the $\alpha$ and $\beta$ were predicted using an approximation of the Newton-Raphson method [30] as: $\hat{\alpha} \approx \frac{3-s \sqrt{(3-s)^{2}+24 s}}{12 s}$, where: $s=\ln \left(\frac{1}{N} \sum_{i=1}^{N} \sigma_{i}^{2}\right)-\frac{1}{N} \sum_{i=1}^{N} \ln \left(\sigma_{i}^{2}\right) ; \hat{\beta}=\frac{\hat{a}}{\mu_{\sigma_{i}^{2}}}$, where: $\mu_{\sigma_{i}^{2}}$ is the mean of estimated $\mathrm{V}_{\mathrm{A}}$ explained by the SNPs. For the haplotyped loci, the estimated $V_{A}$ were dependent on the number of alleles at each haplotyped locus. Therefore, a cubic regression model was used to predict $\alpha$ as a function of the number of alleles. For the $\hat{\beta}$ parameter, the Brody non-linear regression model [31] was applied, as follows: $\hat{\beta}=A\left(1-B e^{-k t}\right)+\varepsilon$, where: $\hat{\beta}$ is a predicted $\beta$ parameter; $A$ is the asymptotic limit for the $\beta$ parameter; $B$ is the integration constant; $k$ is the curve parameter representing the ratio of maximum growth rate to asymptotic limit of $\hat{\beta} ; t$ is the number of alleles at the haplotyped locus; and $\varepsilon$ is the residual. From these equations, it was possible to estimate the gamma distribution in order to establish the thresholds that allowed identifying haplotyped loci (regarding the number of alleles) and SNP markers that explained the greatest amounts of $\mathrm{V}_{\mathrm{A}}$ for WBSF.

\section{Linkage disequilibrium analysis}

The LD between each pair of the SNPs $(413,355)$, measured as $r^{2}$, was calculated using Haploview [32]. The average $r^{2}$ values according to distance between markers are displayed in Fig. 1. The regions that explained the greatest amounts of $\mathrm{V}_{\mathrm{A}}$ for WBSF were classified as strong $\left(\mathrm{r}^{2}>0.6\right)$, moderate $\left(0.2<\mathrm{r}^{2}<0.6\right)$, weak $\left(0.1<\mathrm{r}^{2}<0.2\right)$, and not in $\operatorname{LD}\left(\mathrm{r}^{2}<0.1\right)$ based on the average $\mathrm{r}^{2}$ values between the SNPs.

\section{SNP and haplotype annotation and gene networks}

The SNPs and the genomic regions harboring all of the haplotyped loci identified were annotated using the Variant Effect Predictor (VEP) Ensembl API [33]. The identified genes were used to predict a gene interaction network using the GeneMANIA Cytoscape plug-in [34], based on the source organism Homo sapiens.

\section{Results}

\section{Genome-wide association analysis}

The single-SNP GWAA identified eight variants influencing WBSF on BTA 3, 4, 9, 10 and 11 (Table 1). The SNP rs134499129 (BTA3) explained the greatest amount of $\mathrm{V}_{\mathrm{A}}$ $\left(0.072 \mathrm{~kg}^{2}\right)$ and $r s 41623448$ (BTA10) had the largest allele substitution effect $(0.73 \pm 0.09 \mathrm{~kg})$. Four of the detected variants (rs109294639, rs134499129, rs41595711 and rs42732955), located in NOS1AP and SUCLG1 were 


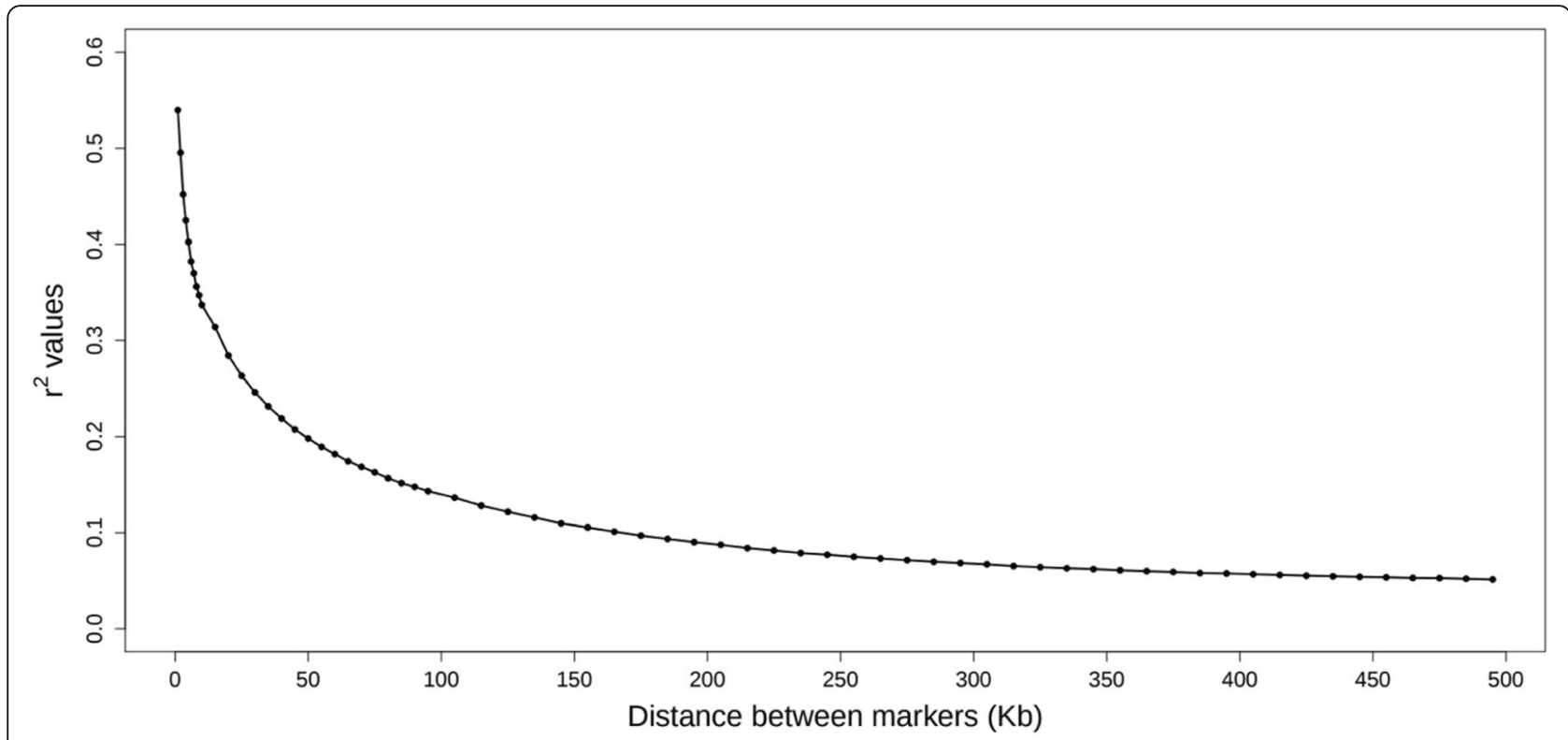

Fig. 1 Linkage disequilibrium $\left(r^{2}\right)$ values according to distance between pairs of markers

intronic, and four variants (rs43490295, rs137367597, rs41623448 and rs136174419) were intergenic. The intronic variant $r s 109294639$ was in moderate LD with rs134499129 and rs41595711 $\left(\mathrm{r}^{2}=0.26\right.$ and 0.24 , respectively), whereas $r s 134499129$ and $r s 41595711$ were in strong LD $\left(r^{2}=0.71\right)$. The intergenic variants $r s 41623448$ and rs136174419 are located near TBCD21 and SUCLG1, at a distance of 37 and $68 \mathrm{~kb}$, respectively. The SNPs rs136174419 (near SUCLG1) and rs42732955 (SUCLG1, intron 1) were in strong LD $\left(\mathrm{r}^{2}=0.79\right)$ at a distance of 74 kb. However, rs43490295, rs137367597 and rs41623448 are not in LD with any neighboring SNP markers.

Using the haplotype-based analysis we identified haplotyped loci that explained the greatest amounts of $\mathrm{V}_{\mathrm{A}}$ for WBSF (Table 2). Thirty-three putative QTL regions for WBSF were detected, with 19 being identified by at least two different haplotype-based analyses and five QTLs were identified in all of the haplotype-based analyses. The
SW3, SW5, SW7, SW9 and SW11 analyses identified 57, $61,42,39$, and $21 \%$ of all 33 putative QTL regions, respectively, whereas, the analyses using SW3 and SW5 jointly detected $88 \%$ of all 33 QTL regions. Increasing the number of SNPs included in the haplotyped loci did not always lead to an increase in the amount of $\mathrm{V}_{\mathrm{A}}$ that was explained by the haplotypes. Some QTLs appeared to only be captured using larger haplotypes while other QTLs could only be detected using smaller haplotypes.

Putative QTLs identified are located on BTA 1, 3, 4, 5, $8,9,10,11,15,17,18,24,25,26$ and 29. SNPs within most of the QTL regions found in this study were in strong or medium pair-wise LD and the length of the QTL regions varied from 5 to $147 \mathrm{~kb}$ with an avarage of $63 \mathrm{~kb}$, as shown in Table 2. The QTL with the largest effect was identified in all of the haplotype window size analyses, however, the window for the SW3 analysis explained the greatest amount of $\mathrm{V}_{\mathrm{A}}\left(0.098 \mathrm{~kg}^{2}\right)$. This

Table 1 SNP markers that explained the greatest additive genetic variance for meat tenderness in Nelore cattle

\begin{tabular}{lllllll}
\hline SNP marker & BTA & Position (bp) & Allele frequencies (effects $\left.{ }^{\mathrm{a}}\right)$ & & Var (kg ${ }^{2}$ ) & Gene \\
\hline rs109294639 & 3 & $7,384,182$ & $T=0.094(-0.40 \pm 0.08)$ & $\mathrm{C}=0.906(0.40 \pm 0.08)$ & 0.027 & NOS1AP (intron1) \\
rs134499129 & 3 & $7,390,035$ & $\mathrm{~A}=0.163(-0.51 \pm 0.06)$ & $\mathrm{G}=0.837(0.51 \pm 0.06)$ & 0.072 & NOS1AP (intron1) \\
rs41595711 & 3 & $7,391,544$ & $T=0.185(-0.30 \pm 0.06)$ & $\mathrm{C}=0.815(0.30 \pm 0.06)$ & 0.027 & NOS1AP (intron1) \\
rs43490295 & 4 & $1,008,553$ & $\mathrm{G}=0.120(-0.37 \pm 0.07)$ & $\mathrm{A}=0.880(0.37 \pm 0.07)$ & 0.028 & - \\
rs137367597 & 9 & $1,116,610$ & $\mathrm{C}=0.135(-0.34 \pm 0.07)$ & $\mathrm{A}=0.865(0.34 \pm 0.07)$ & 0.028 & - \\
rs41623448 & 10 & $20,486,971$ & $\mathrm{C}=0.064(-0.73 \pm 0.09)$ & $T=0.936(0.73 \pm 0.09)$ & 0.063 & near TBCD21 \\
rs136174419 & 11 & $50,332,078$ & $\mathrm{~A}=0.112(-0.37 \pm 0.07)$ & $\mathrm{G}=0.888(0.37 \pm 0.07)$ & 0.028 & near SUCLG1 \\
rs42732955 & 11 & $50,406,682$ & $\mathrm{~A}=0.124(-0.39 \pm 0.07)$ & $\mathrm{G}=0.876(0.39 \pm 0.07)$ & 0.032 & SUCLG1 (intron1) \\
\hline SNP & & & & &
\end{tabular}

SNP single nucleotide polymorphism, BTA Bos taurus autosome, Var SNP marker additive genetic variance

${ }^{a}$ Allele substitution effects from GEMMA software $(\mathrm{kg})$ 
Table 2 QTL regions for meat tenderness detected using haplotype-based analysis on variable-sized sliding windows

\begin{tabular}{|c|c|c|c|c|c|c|c|c|c|c|}
\hline \multirow[t]{2}{*}{ BTA } & \multirow[t]{2}{*}{ Region } & \multirow[t]{2}{*}{ Position (bp) } & \multirow[t]{2}{*}{ Dist } & \multirow[t]{2}{*}{ LD } & \multicolumn{6}{|c|}{ Additive genetic variance $\left(\mathrm{kg}^{2}\right)$} \\
\hline & & & & & SNP & SW3 & SW5 & SW7 & SW9 & SW11 \\
\hline 1 & rs43761056 - rs137207255 & $6,317,864-6,324,488$ & 6625 & M & - & 0.032 & 0.036 & - & - & - \\
\hline 1 & rs42493480 - rs42493494 & $125,981,534-126,011,455$ & 29,922 & M & - & - & 0.031 & 0.034 & 0.038 & - \\
\hline 3 & rs109872503 - rs110244139 & $7,371,235-7,460,489$ & 89,255 & M & 0.072 & 0.098 & 0.071 & 0.085 & 0.086 & 0.089 \\
\hline 3 & rs132795858 - rs134534136 & $29,474,206-29,610,884$ & 136,679 & S & - & 0.038 & 0.049 & 0.051 & 0.051 & 0.050 \\
\hline 3 & rs132763845 - rs109427593 & $90,594,180-90,664,268$ & 70,089 & M & - & - & - & 0.036 & 0.045 & - \\
\hline 3 & rs $134111725-r s 133976586$ & $93,949,186-94,067,553$ & 118,368 & S & - & - & - & - & - & 0.040 \\
\hline 4 & rs43490295 & $1,008,553$ & - & $\mathrm{N}$ & 0.028 & - & - & - & - & - \\
\hline 4 & rs137252969 - rs43385178 & $24,143,662-24,225,606$ & 81,945 & W & - & - & 0.037 & - & - & - \\
\hline 4 & rs $110513194-r s 135604613$ & $27,834,564-27,894,006$ & 59,443 & $S$ & - & - & - & - & 0.042 & - \\
\hline 5 & rs136407209 - rs110430223 & $7,525,121-7,583,435$ & 58,315 & $S$ & - & - & 0.030 & - & - & - \\
\hline 5 & rs41587994 - rs109349300 & $18,434,417-18,465,179$ & 30,763 & $S$ & - & 0.036 & 0.034 & - & - & - \\
\hline 8 & rs 133253155 - rs 135484797 & $92,216,117-92,289,515$ & 73,399 & S & - & 0.033 & 0.034 & 0.033 & - & - \\
\hline 9 & rs137367597 & $1,116,610$ & - & $\mathrm{N}$ & 0.028 & - & - & - & - & - \\
\hline 9 & rs 110938040 - rs 135589084 & $12,339,368-12,406,450$ & 67,083 & M & - & - & - & - & 0.048 & 0.053 \\
\hline 9 & rs $136867942-r s 135252570$ & $21,441,424-21,497,606$ & 56,183 & M & - & - & - & - & 0.037 & - \\
\hline 9 & rs133390891 - rs43600182 & $61,168,282-61,197,874$ & 29,593 & $S$ & - & - & 0.037 & - & - & - \\
\hline 9 & rs $110730224-r s 109847831$ & $66,977,872-67,045,614$ & 67,743 & M & - & 0.040 & 0.039 & 0.040 & 0.040 & - \\
\hline 9 & rs135160781 - rs110936646 & $100,716,451-100,721,452$ & 5002 & $S$ & - & 0.034 & - & - & - & - \\
\hline 10 & rs 137812088 - rs 133615734 & $20,448,593-20,541,764$ & 93,172 & M & 0.063 & 0.040 & 0.041 & 0.038 & 0.044 & 0.052 \\
\hline 10 & rs $134006987-r s 136312573$ & $43,692,759-43,726,489$ & 33,731 & S & - & 0.033 & 0.031 & - & - & - \\
\hline 11 & rs109122230 - rs41655045 & $5,751,331-5,768,629$ & 17,318 & M & - & 0.033 & - & - & - & - \\
\hline 11 & rs43755797 - rs137032372 & $43,129,117-43,135,745$ & 6629 & M & - & 0.032 & - & - & - & - \\
\hline 11 & rs136174419 - rs42731923 & $50,332,078-50,417,494$ & 85,417 & S & 0.032 & 0.069 & - & - & - & - \\
\hline 15 & rs132639440 - rs136091960 & $72,439,829-72,470,564$ & 30,736 & M & - & - & 0.036 & 0.040 & 0.051 & - \\
\hline 17 & rs110304377 - rs42926409 & $70,788,438-70,935,589$ & 147,152 & M & - & 0.035 & 0.043 & 0.054 & 0.056 & - \\
\hline 18 & rs137081181 - rs134146295 & $14,849,540-14,991,573$ & 142,034 & $S$ & - & 0.039 & 0.041 & 0.043 & 0.043 & 0.045 \\
\hline 24 & rs135709192 - rs136715705 & $47,570,379-47,596,815$ & 26,437 & $S$ & - & 0.033 & 0.033 & 0.034 & 0.034 & 0.034 \\
\hline 24 & rs110779214 - rs109148899 & $48,585,933-48,632,298$ & 46,366 & $S$ & - & 0.038 & 0.038 & - & - & - \\
\hline 24 & rs136382747- rs135235176 & $55,868,854-55,975,740$ & 106,887 & M & - & - & - & 0.040 & - & - \\
\hline 25 & rs110607501 - rs108984883 & $17,590,025-17,598,054$ & 8030 & $S$ & - & 0.033 & 0.034 & 0.034 & - & - \\
\hline 26 & rs 133176306 - rs109605337 & $19,035,737-19,066,768$ & 31,032 & S & - & 0.034 & 0.038 & - & - & - \\
\hline 28 & rs134774253 - rs42146826 & $26,876,269-26,885,074$ & 8806 & $S$ & - & 0.033 & - & - & - & - \\
\hline 29 & rs136755211 - rs42192064 & $43,980,089-44,042,363$ & 62,275 & S & - & - & 0.032 & 0.032 & - & - \\
\hline
\end{tabular}

BTA Bos taurus autosome, Dist distance, LD linkage disequilibrium, SNP single nucleotide polymorphism, SW Sliding window haplotype, SW3 SW of three SNPs, SW5 SW of five SNPs, SW7 SW of seven SNPs, SW9 Haplotype SW of nine SNPs, SW11 SW of eleven SNPs, S strong $L D\left(r^{2}>0.6\right), M$ moderate $L D\left(0.2<r^{2}<0.6\right), W$ weak LD $\left(0.1<r^{2}<0.2\right), N$ not LD $\left(r^{2}<0.1\right)$

QTL region harbors NOS1AP (BTA3) and includes three SNPs (rs109294639, rs134499129 and rs41595711) identified in the single-SNP GWAA.

The SNPs rs43490295 and rs137367597 on BTA4 and BTA9 (Table 2), respectively, were only detected by the single-SNP GWAA. On the other hand, the haplotype-based association analyses captured many QTL regions that were not identified by the single-SNP GWAA. Full results for single-SNP and haplotype-based association analyses for each number of alleles at haplotyped loci are shown in Additional files 1, 2, 3, and 4.

\section{Gene network analysis}

Figure 2 illustrates the interactions among the genes located in genomic regions detected as being influencing WBSF in this study. The constructed gene interaction network revealed that $66.7 \%$ of the constituant genes are known to be co-expressed in humans. Moreover, 26.7\% 


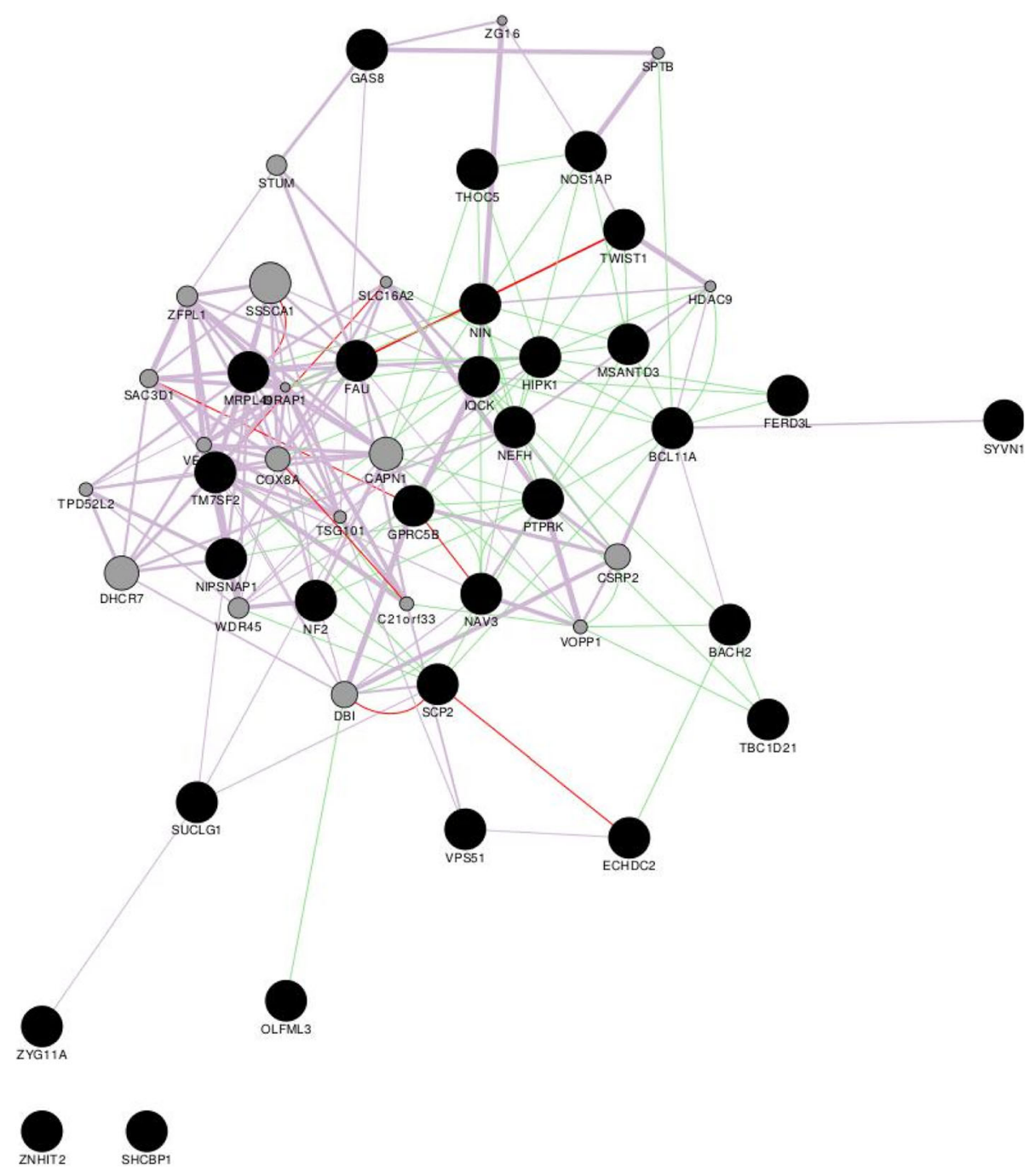

Fig. 2 Gene interaction network for genes in QTL regions for meat tenderness (WBSF). Genes presented as black circles were located in the QTL regions and genes that interact with those as grey circles. Edges in purple, green and red represent co-expression relationships, genetic interactions and co-localizations, respectively

of the genes co-localize indicating that they are expressed in the same tissue or that their proteins are both identified in the same cellular locations. In addition, $70 \%$ of the genes interact, suggesting that they are functionally associated. The gene interaction network also revealed 20 genes, presented as grey circles, that interact with genes in the genomic regions that cause variation in WBSF. Among these, CAPN1 is related to genes that were found to be influencing WBSF in this study. CAPN1 interacts with NEFH, NIN, PTPRK and THOC5 and is co-expressed with MRPL49, SUCLG1, TM7SF2 and NF2.

\section{Discussion}

We performed a single-SNP GWAA and investigated a strategy for haplotype-based analysis using variable-sized sliding windows to detect genomic regions that influence WBSF. The desirable alleles (negative effects) for all eight SNPs identified influencing WBSF using single-SNP GWAA were in low frequency (Table 1), indicating that selection for these desirable alleles could improve tenderness in Nelore cattle. We found haplotypes affecting WBSF that were not detected in the single-SNP analysis (Table 2). This is consistent with previous studies that have shown that haplotype-based analysis provides a greater power for QTL detection than does single SNP analysis $[15,16,35,36]$. The most likely reason for this finding is that QTL are more likely to be in strong LD with a multi-marker haplotype than with a single biallelic SNP, thus haplotype-based association methods have the opportunity to capture greater numbers of associations [10,37, 38]. 


\section{Genome-wide association analysis}

The single-SNP analysis detected two SNPs (rs43490295 and rs137367597) that were not identified in the haplotype-based analyses. These SNPs explained the smallest amounts of QTL variance among the detected SNPs $\left(0.028 \mathrm{~kg}^{2}\right)$ and they were not in LD with neighboring SNPs. Using simulated data, [21] showed that haplotypes did not provide an advantage for detecting QTL with small effect sizes. In addition, haplotype-based analysis may also not have captured these signals because haplotype-based tests tend to be more powerful when moderate to high levels of LD exist in a chromosome region [39].

The variable-sized sliding window haplotype analysis strategy was used to ensure that every region of the genome was included in the analysis and that causal loci could be spanned by haplotyped regions. However, the number of contiguous SNPs to include in a haplotype window is a variable that requires optimization $[9,10]$. The optimal haplotype size will vary according to SNP density, the patterning of LD throughout the genome and the genetic architecture of trait variation [40, 41]. We investigated five window sizes for haplotypes, containing 3, 5, 7, 9 or 11 SNP markers (SW3, SW5, SW7, SW9 and SW11, respectively). The choice of a maximum window length of 11 SNP markers was made based upon computational efficiency. The SW5 analysis detected the largest number of haplotypes loci followed by the SW3 analysis. This result may be because longer haplotype windows are more likely to introduce analytical problems such as high rates of recombination among distal SNPs resulting in excessive numbers of haplotypes, creating noise and computer memory problems [39, 42], and reducing the benefits of improved LD between haplotypes and causal variants [43].

Grapes et al.[35, 44] also found that the power to detect QTL improved as haplotype length increased to 6 SNP markers and decreased thereafter using a simulated bovine data set. However, [45] has shown that haplotypic diversity was best captured by a 20-marker sliding window in U.S. Angus cattle. The extent of LD in Nelore is much less than in Angus [46], suggesting that smaller window sizes will optimally capture haplotypic diversity in indicine cattle breeds. This assumption is supported by the length of all haplotype windows found in this study that ranged from $5 \mathrm{~kb}$ to $147 \mathrm{~kb}$, and the SNPs within most of the QTL regions were in strong or medium pair-wise LD (Table 2). As shown in Fig. 1, the useful LD in our population extends for less than $100 \mathrm{~kb}$ $\left(r^{2}<0.15\right)$, which is consistent with the findings of [47]. This suggests that haplotypes based on sliding windows of size no more than $100 \mathrm{~kb}$ will capture the LD genomewide. On the other hand, some putative QTLs were detected only by larger haplotypes. One possible explanation is that such QTL have small to very small effects on WBSF, therefore many informative SNPs grouped would have larger aggregated effects [22]. Other hypothesis is that only the allele frequencies of the larger haplotypes were similar to the QTL allele frequencies in these regions, which allowed their detection [21, 38]. Thus, since the genetic architecture and population history differ across genes and traits, it is not reasonable to expect that one single method would be superior at detection of all QTL [21].

\section{Genes influencing WBSF}

The SNP and haplotype-based association analyses identified 37 candidate genes influencing WBSF in Nelore cattle (Table 3). Among these, five genes (GAS8, OLFML3, TWIST1, GPRC5B, and HIPK1) are involved in myogenesis, which is responsible for generating muscle tissue during embryonic development, regeneration of the mature skeletal musculature and maintenance of tissue homeostasis [48-52].

Several genes located in the QTL regions for WBSF, such as NEFH, FERD3L, NAV3, BCL11A, ZNHIT2, NOS1AP and SHCBP1, participate in neurogenesis processes (GO:0022008) or the structure and function of neurons. Neurogenesis is essential for skeletal muscle development and regeneration [53]. Motor neuron, a neuromuscular junction component, regulates skeletal muscle contraction [54] and also has a role in the development and differentiating of muscle fibers [55]. NEFH, SHCBP1 and NOS1AP have previously been associated with WBSF in Nelore steers [6]. Moreover, NOS1AP has been associated with longissimus muscle area and marbling score in cattle [56]. BCL11A has been associated with marbling score in Canchim beef cattle [57] and a QTL region for meat tenderness harbored ZNHIT2 in pigs [58].

The NIN and NF2 genes putatively affect skeletal muscle structure and composition. NIN encodes a microtubule nucleation protein, which has previously been associated with skeletal muscle differentiation [59]. NF2 participates in actin cytoskeleton organization (GO:0030036), which may explain the association with WBSF after 7 days of aging in Nelore steers found by [6]. This process results in the assembly, arrangement of constituent parts, or disassembly of cytoskeletal structures comprising actin filaments and their associated proteins (GO:0030036). Actin is a myofibril protein, one of the major components of the sarcomere, which has been reported to affect meat tenderness [60].

Genes involved in lipid and fatty acid metabolism, such as SUCLG1, THOC5, NIPSNAP1, IQCK, TM7SF2, VPS51, PTPRK, ECHDC2 and SCP2, were also found to influence meat tenderness. Positive effects of lipid on meat tenderness are likely due to the lipid within cells in the perimysium, which separate muscle fiber bundles [61]. Furthermore, [62] found a genetic correlation between 
Table 3 Genes located in QTL regions for meat tenderness in Nelore cattle

\begin{tabular}{|c|c|c|c|c|}
\hline$\overline{B T A}$ & Position (bp) & Genes & Gene Name & Gene type \\
\hline 1 & $6,317,864-6,324,488$ & - & - & - \\
\hline 1 & $125,981,534-126,011,455$ & - & - & - \\
\hline 3 & $7,371,235-7,460,489$ & ENSBTAG00000010158 & NOSIAP & Protein coding \\
\hline \multirow[t]{3}{*}{3} & $29,474,206-29,610,884$ & ENSBTAG00000024319 & LOC107132293 & Pseudogene \\
\hline & & ENSBTAG00000011322 & HIPK1 & Protein coding \\
\hline & & ENSBTAG00000011327 & OLFML3 & Protein coding \\
\hline 3 & $90,594,180-90,664,268$ & - & - & - \\
\hline \multirow[t]{3}{*}{3} & $93,949,186-94,067,553$ & ENSBTAG00000002910 & ECHDC2 & Protein coding \\
\hline & & ENSBTAG00000003746 & $S C P 2$ & Protein coding \\
\hline & & ENSBTAG00000026032 & FERD3L & Protein coding \\
\hline 4 & $1,008,553$ & - & - & - \\
\hline 4 & $24,143,662-24,225,606$ & ENSBTAG00000010168 & - & - \\
\hline \multirow[t]{2}{*}{4} & $27,834,564-27,894,006$ & ENSBTAG00000039955 & ZYG11A & Protein coding \\
\hline & & ENSBTAG00000046922 & TWIST1 & Protein coding \\
\hline 5 & $7,525,121-7,583,435$ & ENSBTAG00000009852 & NAV3 & Protein coding \\
\hline 5 & $18,434,417-18,465,179$ & - & - & - \\
\hline 8 & $92,216,117-92,289,515$ & - & - & - \\
\hline 9 & $1,116,610$ & - & - & - \\
\hline 9 & $12,339,368-12,406,450$ & - & - & - \\
\hline 9 & $21,441,424-21,497,606$ & - & - & - \\
\hline 9 & $61,168,282-61,197,874$ & ENSBTAG00000020713 & $\mathrm{BACH} 2$ & Protein coding \\
\hline 9 & $66,977,872-67,045,614$ & ENSBTAG00000020829 & PTPRK & Protein coding \\
\hline 9 & $100,716,451-100,721,452$ & - & - & - \\
\hline 10 & $20,448,593-20,541,764$ & ENSBTAG00000007800 & TBC1D21 & Protein coding \\
\hline 10 & $43,692,759-43,726,489$ & ENSBTAG00000020281 & NIN & Protein coding \\
\hline \multirow[t]{2}{*}{11} & $5,751,331-5,768,629$ & ENSBTAG00000042179 & U6 & SnRNA \\
\hline & & ENSBTAG00000046971 & LOC107131213 & Protein coding \\
\hline 11 & $43,129,117-43,135,745$ & ENSBTAG00000016534 & $B C L 11 A$ & Protein coding \\
\hline \multirow[t]{2}{*}{11} & $50,332,078-50,417,494$ & ENSBTAG00000006075 & SUCLG1 & Protein coding \\
\hline & & ENSBTAG00000042444 & U6 & SnRNA \\
\hline 15 & $72,439,829-72,470,564$ & ENSBTAG00000037580 & MSANTD3 & Protein coding \\
\hline \multirow[t]{4}{*}{17} & $70,788,438-70,935,589$ & ENSBTAG00000013150 & THOC5 & Protein coding \\
\hline & & ENSBTAG00000013153 & NF2 & Protein coding \\
\hline & & ENSBTAG00000013152 & NIPSNAP1 & Protein coding \\
\hline & & ENSBTAG00000013147 & NEFH & Protein coding \\
\hline \multirow[t]{4}{*}{18} & $14,849,540-14,991,573$ & ENSBTAG00000007096 & GAS8 & Protein coding \\
\hline & & ENSBTAG00000025283 & LOC101904595 & Pseudogene \\
\hline & & ENSBTAG00000029640 & U1 & SnRNA \\
\hline & & ENSBTAG00000033441 & SHCBP1 & Protein coding \\
\hline 24 & $47,570,379-47,596,815$ & - & - & - \\
\hline 24 & $48,585,933-48,632,298$ & - & - & - \\
\hline 24 & $55,868,854-55,975,740$ & - & - & - \\
\hline \multirow[t]{2}{*}{25} & $17,590,025-17,598,054$ & ENSBTAG00000019596 & GPRC5B & Protein coding \\
\hline & & ENSBTAG00000044092 & IQCK & Protein coding \\
\hline
\end{tabular}


Table 3 Genes located in QTL regions for meat tenderness in Nelore cattle (Continued)

\begin{tabular}{lllll}
\hline BTA & Position $(\mathrm{bp})$ & Genes & Gene Name & Gene type \\
\hline 26 & $19,035,737-19,066,768$ & - & - & - \\
28 & $26,876,269-26,885,074$ & - & TM7SF2 & - \\
29 & $43,980,089-44,042,363$ & ENSBTAG00000005069 & ZNHIT2 & Protein coding \\
& ENSBTAG00000005073 & MRPL49 & Protein coding \\
& ENSBTAG00000005075 & SYVN1 & Protein coding \\
& ENSBTAG00000005076 & VPS51 & Protein coding \\
& ENSBTAG00000015499 & FAU & Protein coding \\
& ENSBTAG00000020807 & &
\end{tabular}

meat tenderness and fatty acid abundance using animals from the same population as used in the present study. THOC5 was found to be differentially expressed between low and high marbling beef cattle in a longissimus dorsi muscle transcriptome analysis [63]. [58] reported that TM7SF2 and VPS51 were located within a QTL region for meat tenderness in pigs. The protein encoded by PTPRK has been associated with marbling in beef cattle [64]. ECHDC2 expression is negatively correlated with non-esterified fatty acid abundance in pigs [65]. SCP2 was down-regulated in pork with high intramuscular fat content [66] and was associated with WBSF after 7 and 14 days of aging in Nelore steers [6].

There was no evidence of a biological link or biological mechanism connecting TBC1D21, MSANTD3, MRPL49, SYVN1, FAU, ZYG11A or BACH2 genes to meat tenderness. These genes may play a role in the regulation of transcription or translation, or may interact with important genes for meat tenderness as shown in Fig. 2. TBC1D21 encodes a GTPase-activating protein (GO:0090630) for Rab family proteins, which are involved in spermatogenesis [67]. Its association with meat tenderness appears to be through interactions with $B A C H 2$ and PTPRK. MSANTD3 is a member of the MSANTD3 family which contains DNA binding domains for Myb proteins and the SANT domain family. [68] speculated that MSANTD3 may be a transcription factor. In addition, MSANTD3 interacts with BCL11A, NIN, NOS1AP, SCP2 and TWIST1. A QTL region for meat tenderness in pigs harbors MRPL49, SYVN1 and FAU [58]. ZYG11A encodes a protein that plays an important role in the regulation of ubiquitinprotein transferase activity (GO:0051438). A ZYG11A family member has been related to marbling in beef cattle [63]. $\mathrm{BACH} 2$ is a transcription repressor and plays essential roles in the regulation of $\mathrm{B}$ cell development. B cells function in the humoral immunity component of the adaptive immune system by secreting antibodies [69]. $B A C H 2$ has been related to intramuscular fat content in bulls [70] and was associated with WBSF in Nelore steers [6].

\section{Gene network analysis}

The gene network analysis (Fig. 2) revealed interactions among the genes within QTL regions for WBSF and other genes that were not detected as influencing WBSF in the present study (grey circles), which are enriched for their functions in the regulation of transcription (GO:0006355), cell differentiation (GO:0030154), lipid metabolic process (GO:0006629), regulation of angiogenesis (GO:0045766), thyroid hormone transport (GO:0070327), proteolysis (GO:0006508), cellular response to insulin stimulus (GO:0032869) and cytoskeleton organization (GO: 0007010). Among these, is a well-known gene influencing WBSF, CAPN1, which is responsible for the postmortem breakdown of myofibrillar proteins and seems to be the primary enzyme involved in the postmortem tenderization process [71]. The CAPN1 interacts with TM7SF2, ZNHIT2, MRPL49, SYVN1, VPS51 and FAU genes that are located in a QTL region for meat tenderness in this study. In addition, all of these genes are located near CAPN1 on BTA29 and their SNPs are in strong LD with SNPs located in CAPN1 in this population (with maximum $\mathrm{r}^{2}=0.86$ ).

\section{Conclusions}

This study demonstrates that GWAA using haplotypes based on variable-sized sliding windows provides substantially more power to detect QTL than does single-SNP analysis, suggesting that this methodology should be considered for genomic predictions for WBSF and other traits. Analyses performed with smaller haplotype windows (3 and 5 SNPs) detected a higher proportion of QTLs than the analyses that used larger SNP windows. However, no single sliding window analysis identified all of the QTL that were found in the analyses using window sizes from 3 to 11 SNPs. This suggests that haplotype-based GWAA should employ several window sizes in order to detect the largest number of putative QTL. Likewise, the single-SNP analysis found two putative QTL that were not found by the haplotype-based analyses. While these may be type I errors, they may also be regulatory variants. 
We identified thirty-seven candidate genes influencing meat tenderness that participate in myogenesis, neurogenesis, lipid and fatty acid metabolism and skeletal muscle structure or composition processes. These findings contribute to a better understanding of the biological mechanisms underlying meat tenderness in Nelore cattle. Further validation of these genes and polymorphisms in different populations would contribute to their use in breeding programs for Nelore cattle.

\section{Additional files}

Additional file 1: Manhattan plot for single-SNP genome-wide association analysis. (PDF $55 \mathrm{~kb}$ )

Additional file 2: $\mathrm{QQ}$ plot for single-SNP genome-wide association analysis. (PDF $14 \mathrm{~kb}$ )

Additional file 3: Manhattan plots for haplotype-based association analyses for each number of alleles at haplotyped loci. (PDF $8648 \mathrm{~kb}$ )

Additional file 4: $\mathrm{QQ}$ plots for haplotype-based association analyses for each number of alleles at haplotyped loci. (PDF $2144 \mathrm{~kb}$ )

\section{Abbreviations}

SNP: Single nucleotide polymorphism; GWAA: Genome-wide association analysis; LD: Linkage disequilibrium; QTL: Quantitative trait locus;

CG: Contemporary group; WBSF: Warner-Bratzler shear force; SW3: Sliding window haplotypes of three SNPs; SW5: Sliding window haplotypes of five SNPs; SW7: Sliding window haplotypes of seven SNPS; SW9: Sliding window haplotypes of nine SNPs; SW11: Sliding window haplotypes of eleven SNPS; GRM: Genomic relationship matrix; VEP: Variant effect predictor; BTA: Bos taurus autosome; NO: Nitric-oxide; nNOS: Neuronal nitric oxide synthase; HDL: High-density lipoprotein; GO: Gene ontology

\section{Acknowledgements}

The authors would like to acknowledge the DeltaGen, PAINT and Cia de Melhoramento breeding programs for providing data. We also would like to acknowledge the members of the Animal Breeding and Genetics Postgraduate Program (PPGMA) of the São Paulo State University (FCAV/Unesp) for their assistance with collecting data.

\section{Funding}

This research was supported by the São Paulo Research Foundation (FAPESP) and National Council of Technological and Scientific Development (CNPq) grants: \#2009/16118-5 and \#480369/2009-7, respectively. São Paulo Research Foundation (FAPESP) also funds the scholarships \#2013/00035-9 and 2014/23013-3 to Camila U. Braz.

\section{Availability of data and materials}

The datasets generated and/or analyzed during the current study are not publicly available because they include confidential information from the companies that provided the samples. The datasets supporting the conclusions of this article are included within the article and its additional file.

\section{Authors' contributions}

CUB conceived the study, analyzed and interpreted the results and, wrote the manuscript. TB, RE and FLBF helped conceive the study. JFT, RC, FB and LGA assisted with preparation of the manuscript and aided with interpretation of results; HNO assisted with analyses, preparation of the manuscript and interpretation of results. All authors read and approved the final manuscript.

\section{Ethics approval}

Use of animals was approved by the São Paulo State University (Unesp), School of Agricultural and Veterinary Science Ethical Committee (Approval No. 18.340/16).
Consent for publication

Not applicable.

\section{Competing interests}

The authors declare that they have no competing interests.

\section{Publisher's Note}

Springer Nature remains neutral with regard to jurisdictional claims in published maps and institutional affiliations.

\section{Author details}

${ }^{1}$ Animal Science Department, São Paulo State University (Unesp), Jaboticabal, SP 144884-900, Brazil. ²Division of Animal Sciences, University of Missouri, Columbia, MO 65211, USA.

Received: 9 August 2018 Accepted: 2 January 2019

Published online: 14 January 2019

\section{References}

1. Matukumalli LK, Lawley CT, Schnabel RD, Taylor JF, Allan MF, Heaton MP, et al. Development and characterization of a high density SNP genotyping assay for cattle. PLoS One. 2009:4:e5350

2. Cole JB, Wiggans GR, Ma L, Sonstegard TS, LawlorJr TJ, Crooker BA, et al. Genome-wide association analysis of thirty one production, health, reproduction and body conformation traits in contemporary U.S. Holstein cows. BMC Genomics. 2011:12:408.

3. Berry DP, Crowley JJ. Residual intake and body weight gain: a new measure of efficiency in growing cattle. J Anim Sci. 2012;90:109-15.

4. Bolormaa S, Lr PN, Zhang YD, Bunch RJ, Harrison BE, Goddard ME, et al. A genome-wide association study of meat and carcass traits in Australian cattle. J Anim Sci. 2011;89:2297-309.

5. McClure MC, Ramey HR, Rolf MM, Mckay SD, Decker JE, Chapple RH, et al. Genome-wide association analysis for quantitative trait loci influencing Warner-Bratzler shear force in five taurine cattle breeds. Anim Genet. 2012; 43:662-73.

6. Tizioto PC, Decker JE, Taylor JF, Schnabel RD, Mudadu MA, Silva FL, et al. Genome scan for meat quality traits in Nelore beef cattle. Physiol Genomics. 2013;45:1012-20.

7. Magalhães AFB, de Camargo GMF, Fernandes Junior GA, Gordo DGM, Tonussi RL, Costa RB, et al. Genome-wide association study of meat quality traits in Nellore cattle. PLoS One. 2016;11:e0157845.

8. Mennecke BE, Townsend AM, Hayes DJ, Lonergan SM. A study of the factors that influence consumer attitudes toward beef products using the conjoint market analysis tool. J Anim Sci. 2007:85:2639-59.

9. Tang R, Feng T, Sha Q, Zhang S. A variable-sized sliding-window approach for genetic association studies via principal component analysis. Ann Hum Genet. 2009;73:631-7.

10. Guo Y, Li J, Bonham AJ, Wang Y, Deng H. Gains in power for exhaustive analyses of haplotypes using variable-sized sliding window strategy: a comparison of association-mapping strategies. Eur J Hum Genet. 2009;17:785-92.

11. Morris RW, Kaplan NL. On the advantage of haplotype analysis in the presence of multiple disease susceptibility alleles. Genet Epidemiol. 2002;23:221-33.

12. Clark AG. The role of haplotypes in candidate gene studies. Genet Epidemiol. 2004;27:321-33.

13. Bardel C, Danjean V, Hugot JP, Darlu P, Genin E. On the use of haplotype phylogeny to detect disease susceptibility loci. BMC Genet. 2005;6:24.

14. Li Y, Sung WK, Liu JJ. Association mapping via regularized regression analysis of single-nucleotide-polymorphism haplotypes in variable-sized sliding windows. Am J Hum Genet. 2007:80:705-15.

15. Hayes BJ, Chamberlain AJ, McPartlan H, Macleod I, Ethuraman L, Goddard ME. Accuracy of marker-assisted selection with single markers and marker haplotypes in cattle. Genet Res. 2007;89:215-20.

16. Calus MP, Meuwissen TH, Windig JJ, Knol EF, Schrooten C, Vereijken AL, et al. Effects of the number of markers per haplotype and clustering of haplotypes on the accuracy of QTL mapping and prediction of genomic breeding values. Genet Sel Evol. 2009;41:11.

17. Martin ER, Lai EH, Gilbert JR, Rogala AR, Afshari AJ, Riley J, et al. SNPing away at complex diseases: analysis of single-nucleotide polymorphisms around APOE in Alzheimer disease. Am J Hum Genet. 2000;67:383-94. 
18. Gabriel SB, Schaffner SF, Nguyen H, Moore JM, Roy J, Blumenstiel B, et al. The structure of haplotype blocks in the human genome. Science. 2002;296:2225-9.

19. Zhao HG, Pfeiffer R, Gail MH. Haplotype analysis in population genetics and association studies. Pharmacogenomics. 2003;4:171-8.

20. Cheng R, Ma JZ, Elston RC, Li MD. Fine mapping functional sites or regions from case-control data using haplotypes of multiple linked SNPs. Ann Hum Genet. 2005;69:102-12.

21. Lorenz AJ, Hamblin MT, Jannink J-L. Performance of single nucleotide polymorphisms versus haplotypes for genome-wide association analysis in barley. PLoS One. 2010;5:e14079.

22. Barendse W. Haplotype analysis improved evidence for candidate genes for intramuscular fat percentage from a genome wide association study of cattle. PLoS One. 2011;6:e29601.

23. Wheeler TL, Koohmaraie M, Shackelford SD. Standardized Warner-Bratzler shear force procedures for meat tenderness measurement. Hruska U. S. MARC. USDA: Clay Center: Roman L; 1995.

24. Sargolzaei M, Chesnais JP, Schenkel FS. A new approach for efficient genotype imputation using information from relatives. BMC Genomics. 2014;15:478

25. Carvalheiro R, Boison SA, Neves HHR, Sargolzaei M, Schenkel FS, Utsunomiya YT, O'Brien AMP, Sölkner J, McEwan JC, Van Tassell CP, Sonstegard TS, Garcia JF. Accuracy of genotype imputation in Nelore cattle. GSE. 2014;46:69.

26. Misztal I, Tsuruta S, Strabel T, Auvray B, Druet T, Lee DH. BLUPF90 and related programs (BGF90). In: Proc. 7th world Congr. Genet. Montpellier, France. Communication no: Appl. Livest. Prod; 2002. p. 28-07.

27. Zhou X, Stephens M. Genome-wide efficient mixed-model analysis for association studies. Nat Genet. 2012:44:821-4.

28. Lynch M, Walsh B. Genetics and Analysis of Quantitative Traits. Sunderland: Sinauer; 1998. p. 980

29. Hayes B, Goddard M. The distribution of the effects of genes affecting quantitative traits in livestock. GSE. 2001;33:209-29.

30. Choi SC, Wette R. Maximum likelihood estimation of the parameters of the gamma distribution and their bias. Technometrics. 1969;11:683-90.

31. Brody S. Bioenergetics and growth with special reference to the efficiency complex in domestic animals. New York: Reinhold Publishing Corporation; 1945. p. 1023

32. Barrett JC, Fry B, Maller J, Daly MJ. Haploview: analysis and visualization of LD and haplotype maps. Bioinformatics. 2005;21:263-5.

33. McLaren W, Gil L, Hunt SE, Riat HS, Ritchie GR, Thormann A, Flicek P, Cunningham F. The Ensembl variant effect predictor. Genome Biol. 2016;17:122.

34. Warde-Farley D, Donaldson SL, Comes O, Zuberi K, Badrawi R, Chao P, Franz M, Grouios C, Kazi F, Lopes CT, Maitland A, Mostafavi S, Montojo J, Shao Q, Wright G, Bader GD, Morris Q. The GeneMANIA prediction server: biological network integration for gene prioritization and predicting gene function. Nucleic Acids Res. 2010;38:W214-20.

35. Grapes L, Dekkers JCM, Rothschild MF, Fernando RL. Comparing linkage disequilibrium-based methods for fine mapping quantitative trait loci. Genetics. 2004;166:1561-70.

36. Dikmen S, Cole JB, Null DJ, Hansen PJ. Genome-wide association mapping for identification of quantitative trait loci for rectal temperature during heat stress in Holstein cattle. PLoS One. 2013;8:e69202.

37. Kaplan N, Morris R. Issues concerning association studies for fine mapping a susceptibility gene for a complex disease. Genet Epidemiol. 2001;20:432-57.

38. Cuyabano BCD, Su G, Lund MS. Genomic prediction of genetic merit using LD-based haplotypes in the Nordic Holstein population. BMC Genomics. 2014;15:1171.

39. Kim Y, Feng S, Zeng ZB. Measuring and partitioning the high-order linkage disequilibrium by multiple order Markov chains. Genet Epidemiol. 2008:32:301-12

40. Durrant C, Zondervan KT, Cardon LR, Hunt S, Deloukas P, Morris AP. Linkage disequilibrium mapping via cladistic analysis of single-nucleotide polymorphism haplotypes. Am J Hum Genet. 2004;75:35-43.

41. Beissinger TM, Rosa GJ, Kaeppler SM, Gianola D, de Leon N. Defining window-boundaries for genomic analyses using smoothing spline techniques. Genet Sel Evol. 2015;47:30

42. Dai JY, Leblanc M, Smith NL, Psaty B, Kooperberg C. SHARE: an adaptive algorithm to select the most informative set of SNPs for candidate genetic association. Biostatistics. 2009:10:680-93.

43. Becker $T$, Herold C. Joint analysis of tightly linked SNPs in screening step of genome-wide association studies leads to increased power. Eur J Hum Genet. 2009;17:1043-9.
44. Boleckova J, Christensen OF, Sorensen P, Sahana G. Strategies for haplotypebased association mapping in a complex pedigreed population. J Czech J Anim Sci. 2012;57:1-9

45. Hoff JL, Decker JE, Schnabel RD, Taylor JF. Candidate lethal haplotypes and causal mutations in Angus cattle. BMC Genomics. 2017;18:799.

46. McKay SD, Schnabel RD, Murdoch BM, Matukumalli LK, Aerts J, Coppieters W, et al. Whole genome linkage disequilibrium maps in cattle. BMC Genet. 2007;8:74.

47. Espigolan R, Baldi F, Boligon AA, Souza FR, Gordo DG, Tonussi RL, et al. Study of whole genome linkage disequilibrium in Nellore cattle. BMC Genomics. 2013;14:305.

48. Bentzinger CF, Wang YX, Rudnicki MA. Building muscle: molecular regulation of myogenesis. Cold Spring Harb Perspect Biol. 2012;4: a008342.

49. Lewis WR, Malarkey EB, Tritschler D, Bower R, Pasek RC, Porath JD, et al. Mutation of growth arrest specific 8 reveals a role in motile cilia function and human disease. PLoS Genet. 2016;12:e1006220.

50. Zhao S, Zhang J, Hou X, Zan L, Wang N, Tang Z, et al. OLFML3 expression is decreased during prenatal muscle development and regulated by microRNA-155 in pigs. Int J Biol Sci. 2012:8:459-69.

51. Castanon I, Baylies MK. A twist in fate: evolutionary comparison of twist structure and function. Gene. 2002;287:11-22.

52. Verheyen EM, Gottardi CJ. Regulation of Wnt/B-catenin signaling by protein kinases. Dev Dyn. 2010;239:34-44.

53. Henningsen J, Rigbolt KTG, Blagoev B, Pedersen BK, Kratchmarova I. Dynamics of the skeletal muscle secretome during myoblast differentiation. Mol Cell Proteomics. 2010;9:2482-96.

54. Bloch-Gallego E. Mechanisms controlling neuromuscular junction stability. Cell Mol Life Sci. 2015;72:1029-43.

55. Jellies J. Muscle assembly in simple systems. Trends Neurosci. 1990;13: 126-31.

56. Makina SO, Muchadeyi FC, van Marle-Köster E, Taylor JF, Makgahlela ML, Maiwashe A. Genomewide scan for selection signatures in six cattle breeds in South Africa. Genet Sel Evol. 2015:47:92.

57. Mokry FB, Higa RH, Mudadu MA, Lima AO, Meirelles SL, da Silva MVB, et al. Genome-wide association study for backfat thickness in Canchim beef cattle using random Forest approach. BMC Genet. 2013;14:47.

58. Casiró S, Velez-lrizarry D, Ernst CW, Raney NE, Bates RO, Charles MG, et al. Genome-wide association study in an F2 Duroc x Pietrain resource population for economically important meat quality and carcass traits. J Anim Sci. 2017:95:545-58

59. Bugnard E, Zaal KJ, Ralston E. Reorganization of microtubule nucleation during muscle differentiation. Cell Motil and the Cytoskeleton. 2005;60:1-13.

60. Ertbjerg P, Puolanne E. Muscle structure, sarcomere length and influences on meat quality: a review. Meat Sci. 2017;132:139-52.

61. Wood JD, Richardson RI, Nute GR, Fisher AV, Campo MM, Kasapidou $E$, et al. Effects of fatty acids on meat quality: a review. Meat Sci. $2004 ; 66: 21-32$

62. Feitosa FL, Olivieri BF, Aboujaoude C, Pereira AS, de Lemos MV, Chiaia HL, et al. Genetic correlation estimates between beef fatty acid profile with meat and carcass traits in Nellore cattle finished in feedlot. J Appl Genetics. 2017:58:123.

63. Chen D, Li W, Du M, Wu M, Cao B. Sequencing and characterization of divergent marbling levels in the beef cattle (longissimus dorsi muscle) transcriptome. Asian-Australas J Anim Sci. 2015;28:158-65.

64. Gao H, Wu Y, Li J, Li H, Li J, Yang R. Forward LASSO analysis for high-order interactions in genome-wide association study. Brief Bioinform. 2014;15:552-61.

65. Yang B, Bassols A, Saco Y, Pérez-Enciso M. Association between plasma metabolites and gene expression profiles in five porcine endocrine tissues. Genet Sel Evol. 2011;43:28.

66. Hamill RM, McBryan J, McGee C, Mullen AM, Sweeney T, Talbot A, Cairns MT, Davey GC. Functional analysis of muscle gene expression profiles associated with tenderness and intramuscular fat content in pork. Meat Sci. 2012:92:440-50

67. Nakamura Y, Asano A, Hosaka Y, Takeuchi T, Iwanaga T, Yamano Y. Expression and intracellular localization of TBC1D9, a Rab GTPaseaccelerating protein, in mouse testes. Exp Anim. 2015:64:415-24.

68. Generous A, Thorson M, Barcus J, Jacher J, Busch M, Sleister H. Identification of putative interactions between swine and human influenza a virus nucleoprotein and human host proteins. Virol J. 2014;11:228. 
69. Sasaki S, Ito E, Toki T, Maekawa T, Kanezaki R, Umenai T, et al. Cloning and expression of human B cell-specific transcription factor BACH2 mapped to chromosome 6q15. Oncogene. 2000;19:3739-49.

70. Komolka K, Ponsuksili S, Elke A, Christa K, Wimmers K, Maak S. Gene

expression profile of Musculus longissimus dorsi in bulls of a Charolais $X$ Holstein $\mathrm{F} 2$-cross with divergent intramuscular fat content. Genom Data. 2016;7:131-3

71. Koohmaraie M. Biochemical factors regulating the toughening and tenderization processes of meat. Meat Sci. 1996;43:193-201.

Ready to submit your research? Choose BMC and benefit from:

- fast, convenient online submission

- thorough peer review by experienced researchers in your field

- rapid publication on acceptance

- support for research data, including large and complex data types

- gold Open Access which fosters wider collaboration and increased citations

- maximum visibility for your research: over $100 \mathrm{M}$ website views per year

At $\mathrm{BMC}$, research is always in progress.

Learn more biomedcentral.com/submissions 